\title{
Pegmatitic Nb-Ta oxide minerals in alluvial placers from Limbach, Bratislava Massif, Western Carpathians, Slovakia: compositional variations and evolutionary trend
}

\author{
Pavel UHER ${ }^{1, *}$, Peter ŽITŇAN², Daniel OZDÍN ${ }^{3}$ \\ ${ }^{1}$ Department of Mineral Deposits, Faculty of Natural Sciences, Comenius University, Mlynská dolina, 84215 Bratislava, Slovak \\ Republic; puher@fns.uniba.sk \\ ${ }^{2}$ Eastern Mediterranean Resources, Radničné námestie 13, 96901 Banská Štiavnica, Slovak Republic \\ ${ }^{3}$ Department of Mineralogy and Petrology, Faculty of Natural Sciences, Comenius University, Mlynská dolina, 84215 Bratislava, \\ Slovak Republic \\ *Corresponding author
}

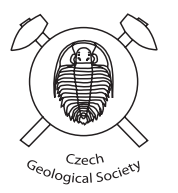

Niobium-tantalum mineral assemblage of granitic pegmatite origin was found in alluvial placers near Limbach, the Bratislava granitic massif, Malé Karpaty Mountains, Central Western Carpathians, southwest Slovakia. The most widespread mineral is ferrocolumbite to ferrotantalite, rarely manganocolumbite to manganotantalite I [at. $\mathrm{Mn} /(\mathrm{Mn}+\mathrm{Fe})=$ $0.17-0.52$ and $\mathrm{Ta} /(\mathrm{Ta}+\mathrm{Nb})=0.19-0.70]$ shows primary magmatic, fine to coarse, regular oscillatory zoning with an evolutionary trend indicating a moderate degree of fractionation, analogous to beryl-columbite subtype of rare-element granitic pegmatites. On the contrary, irregular oscillatory and patchy zoning of ferrocolumbite to manganotantalite II $[\mathrm{Mn} /(\mathrm{Mn}+\mathrm{Fe})=0.17-0.66, \mathrm{Ta} /(\mathrm{Ta}+\mathrm{Nb})=0.16-0.81]$ together with precipitation of anhedral Ta-rich intergrowths and inclusions of ferrotapiolite $[\mathrm{Mn} /(\mathrm{Mn}+\mathrm{Fe}=0.05-0.07, \mathrm{Ta} /(\mathrm{Ta}+\mathrm{Nb})=0.87-0.93], \mathrm{Ta}>\mathrm{Nb}$-rich rutile $[\mathrm{Mn} /(\mathrm{Mn}+\mathrm{Fe})=$ $0.02-0.03, \mathrm{Ta} /(\mathrm{Ta}+\mathrm{Nb})=0.80-0.86]$, Sn-rich ixiolite $[\mathrm{Mn} /(\mathrm{Mn}+\mathrm{Ta})=0.40-0.41, \mathrm{Ta} /(\mathrm{Ta}+\mathrm{Nb})=0.81]$ and uraninite + uranmicrolite $[\mathrm{Ta} /(\mathrm{Ta}+\mathrm{Nb})=0.81-0.94]$ indicates their subsolidus origin.

Keywords: columbite-tantalite, ferrotapiolite, ixiolite, Ta-rich rutile, uranmicrolite, pegmatite.

Received: 19 December 2006; accepted 25 May 2007; handling editor: V. Janoušek

\section{Introduction}

Niobium-tantalum oxide minerals, especially columbite and the ixiolite group, the tapiolite series, and $\mathrm{Nb}, \mathrm{Ta}-$ rich rutile belong to the most characteristic accessory phases of rare-element granitic pegmatites and highly fractionated granites. Their compositional variations within discrete crystals, host-rock zones, localities as well as geological units (provinces) strongly depend on several factors, such as temperature, fractionation level, local composition, $\mathrm{pH}$, fluid activity (especially $\mathrm{F}, \mathrm{H}_{2} \mathrm{O}$ and $\mathrm{B}$ ), $\mathrm{Nb}$ vs. Ta solubility in melt, and crystallo-chemical properties. Consequently, they can serve as sensitive genetic indicators (e.g. Černý et al. 1985, 1986; Černý and Ercit 1989; Linnen and Keppler 1997; Linnen 1998; Tindle and Breaks 2000; Novák et al. 2000, 2003).

The genetic interpretation of $\mathrm{Nb}-\mathrm{Ta}$ minerals in secondary geological occurrences (for example in elluvial to alluvial deposits), where information about the parental pegmatite or granite rock are lost, is less straightforward. However, detailed knowledge of characteristic textural, paragenetic, and compositional relations of the $\mathrm{Nb}-\mathrm{Ta}$ phases in such a secondary geological occurrence could serve as a useful tool for interpretation of the primary character and genetic aspects of their host rocks. Our de- scription of $\mathrm{Nb}$-Ta oxide mineralization in alluvial placers near Limbach in the Bratislava Granitic Massif, Western Carpathians, is one example of such a study.

\section{Regional geology}

The niobium-tantalum oxide minerals were obtained from the heavy-mineral fraction of recent alluvial placers of the Limbach Brook, at Slnečné Valley in the Malé Karpaty Mountains, $3 \mathrm{~km}$ north of the village of Limbach, c. $15 \mathrm{~km}$ north-northeast of Bratislava. The Limbach Brook and its tributaries drain the Staré Mesto granitic body, a part of the Bratislava Granitic Massif (BGM) and also the surrounding metapelites-metapsammites, an area around $4 \mathrm{~km}^{2}$ in size (Fig. 1). The BGM represents orogenic, peraluminous calc-alkaline granitic plutonic suite with S-type affinity (e.g. Cambel and Vilinovič 1987; Petrík et al. eds 2001). The age of the BGM is Hercynian, Lower Carboniferous, c. $350 \mathrm{Ma}$ on the basis of whole-rock $\mathrm{Rb}-\mathrm{Sr}$ isochron (Bagdasaryan et al. 1982) and electron-microprobe $\mathrm{U}-\mathrm{Th}-\mathrm{Pb}$ monazite dating (Uher et al. unpublished data). The granitic rocks exhibit distinct intrusive and thermal metamorphic contact with adjacent Lower Paleozoic metapelites to metapsammites 


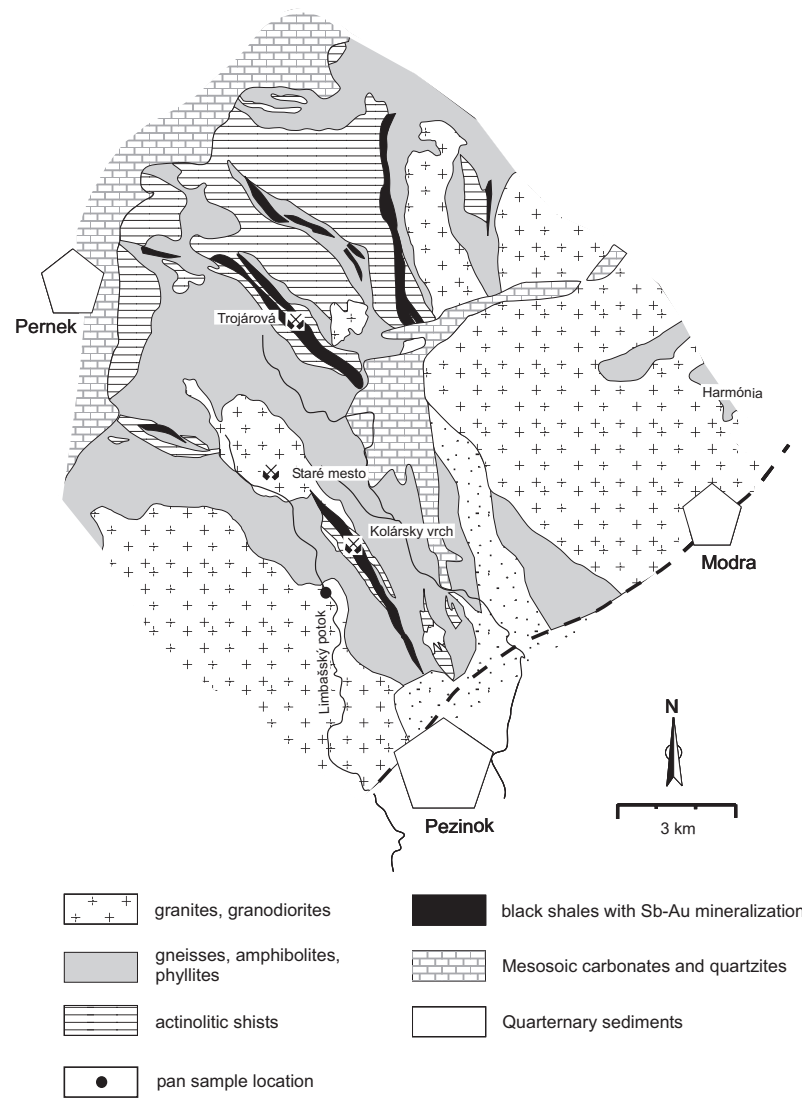

Fig. 1 Generalized geological map of the Limbach area, Malé Karpaty Mountains (modified from Bakos and Chovan eds 2004).

of micaschist to paragneiss character, metamorphosed in amphibolite facies at $\mathrm{P} \leq 300-350 \mathrm{MPa}$ and $\mathrm{T} \leq 550^{\circ} \mathrm{C}$, which corresponds to a depth of granite emplacement of approximately 12 to $14 \mathrm{~km}$ (Korikovsky et al. 1984). Due to the peri-plutonic thermal metamorphism around the BGM, the following metamorphic zones developed in the country-rock metapelites: biotite, garnet, staurolite-chlorite, and staurolite-sillimanite (Korikovsky et al. 1984). The fundamental rock types of the BGM are biotite granodiorites to muscovite-biotite monzogranites, less frequently leucocratic two-mica to muscovite syenogranites and small bodies of biotite-amphibole diorites (Cambel and Vilinovič 1987). The granitic rocks are usually medium-grained and equigranular, rarely porphyritic with K-feldspar phenocrysts. Gold from hydrothermal quartz veins in granites has been exploited from a small deposit in the Staré Mesto area (Bakos and Chovan eds 2004).

Dikes of granitic pegmatites and aplites are widespread in the BGM and also in the Limbach area. The pegmatite dikes, usually up to 1-2 m thick, commonly show zoned internal structure with graphic, blocky K-feldspar, coarse-grained alkali feldspar-quartz-muscovite \pm biotite and blocky quartz core zones, locally with late fan-like muscovite and saccharoidal albite-rich replacement zones (Dávidová 1970, 1978). The most fractionated granitic pegmatites of the BGM contain accessory beryl and columbite-tantalite group minerals (Uher et al. 1994) and they could be classified as belonging to the LCT family, beryl-columbite subgroup of the rare-element class of granitic pegmatites (sensu Černý and Ercit 2005).

\section{Analytical methods}

Electron-microprobe analyses of $\mathrm{Nb}$-Ta oxide minerals were carried out in the wavelength-dispersion mode using a Cameca SX-100 instrument at the State Geological Survey of Slovak Republic, Bratislava, with a beam diameter of $1-2 \mu \mathrm{m}$ and an accelerating voltage of $15 \mathrm{kV}$, a sample current of $20 \mathrm{nA}$, and a counting time of 20 to $40 \mathrm{~s}$. The following standards were used: scheelite $(\mathrm{WM} \beta), \mathrm{LiNbO}_{3}(\mathrm{Nb} L \alpha), \mathrm{LiTaO}_{3}(\mathrm{Ta} M \alpha), \mathrm{TiO}_{2}(\mathrm{TiK} \alpha)$, $\mathrm{SnO}_{2}(\mathrm{Sn} L \alpha), \mathrm{ZrO}_{2}(\mathrm{Zr} L \alpha), \mathrm{UO}_{2}(\mathrm{U} M \beta), \mathrm{ScPO}_{4}(\mathrm{Sc} K \alpha)$, $\mathrm{YPO}_{4}(\mathrm{Y} L \alpha)$, metallic $\mathrm{Sb}(\mathrm{Sb} L \alpha)$, hematite $(\mathrm{Fe} K \alpha)$, rhodonite $(\mathrm{Mn} K \alpha), \mathrm{MgO}(\mathrm{Mg} K \alpha)$, wollastonite $(\mathrm{Ca} K \alpha)$, willemite $(\mathrm{ZnK \alpha})$, and $\mathrm{PbS}(\mathrm{Pb} M \alpha)$. For data processing, PAP corrections were used.

\section{Results}

The $\mathrm{Nb}$-Ta oxide minerals were identified as rare black prismatic crystals and their broken fragments up to $4 \mathrm{~mm}$ in size from the heavy mineral fraction of the sandy alluvium of the Limbach Brook. The crystals are relatively fresh with metallic to semi-metallic luster, without any rounding or other features indicative of a longer transport. The heavy mineral assemblage consists of garnet (almandine-spessartine), staurolite, ilmenite, magnetite, zircon, apatite, monazite and gold.

Minerals of the columbite-tantalite group are the most widespread Nb-Ta phases. Two distinct textural, compositional and genetic types can be distinguished (Figs 2a-f):

(1) Large crystals and fragments (up to $4 \mathrm{~mm}$ ) with regular fine to coarse oscillatory zoning, locally unzoned or with diffuse zoning ( $\boldsymbol{C t}$ I, Fig. 2a). Compositionally they correspond to ferrocolumbite, rarely ferrotantalite, manganocolumbite, and manganotantalite with broad compositional variations (atomic ratios): $\mathrm{Mn} /(\mathrm{Mn}+\mathrm{Fe})$ $=0.17-0.52$ and $\mathrm{Ta} /(\mathrm{Ta}+\mathrm{Nb})=0.19-0.70(\mathrm{Tab} .1$, Figs 3 and 4$)$. The Ti contents are generally low $(0.4-1.3 \mathrm{wt} . \%$ $\mathrm{TiO}_{2}$ ). Locally, $\mathrm{Ct}$ I shows slightly increased $\mathrm{Zr}$ concentrations ( 0.05 to 0.9 wt. $\left.\% \mathrm{ZrO}_{2}\right)$. The contents of other elements (W, Sn, U, Sb and $\mathrm{Zn}$ ) are negligible.

(2) Zones within columbite-tantalite crystals and their fragments with various irregular zoned textures (Ct II). 

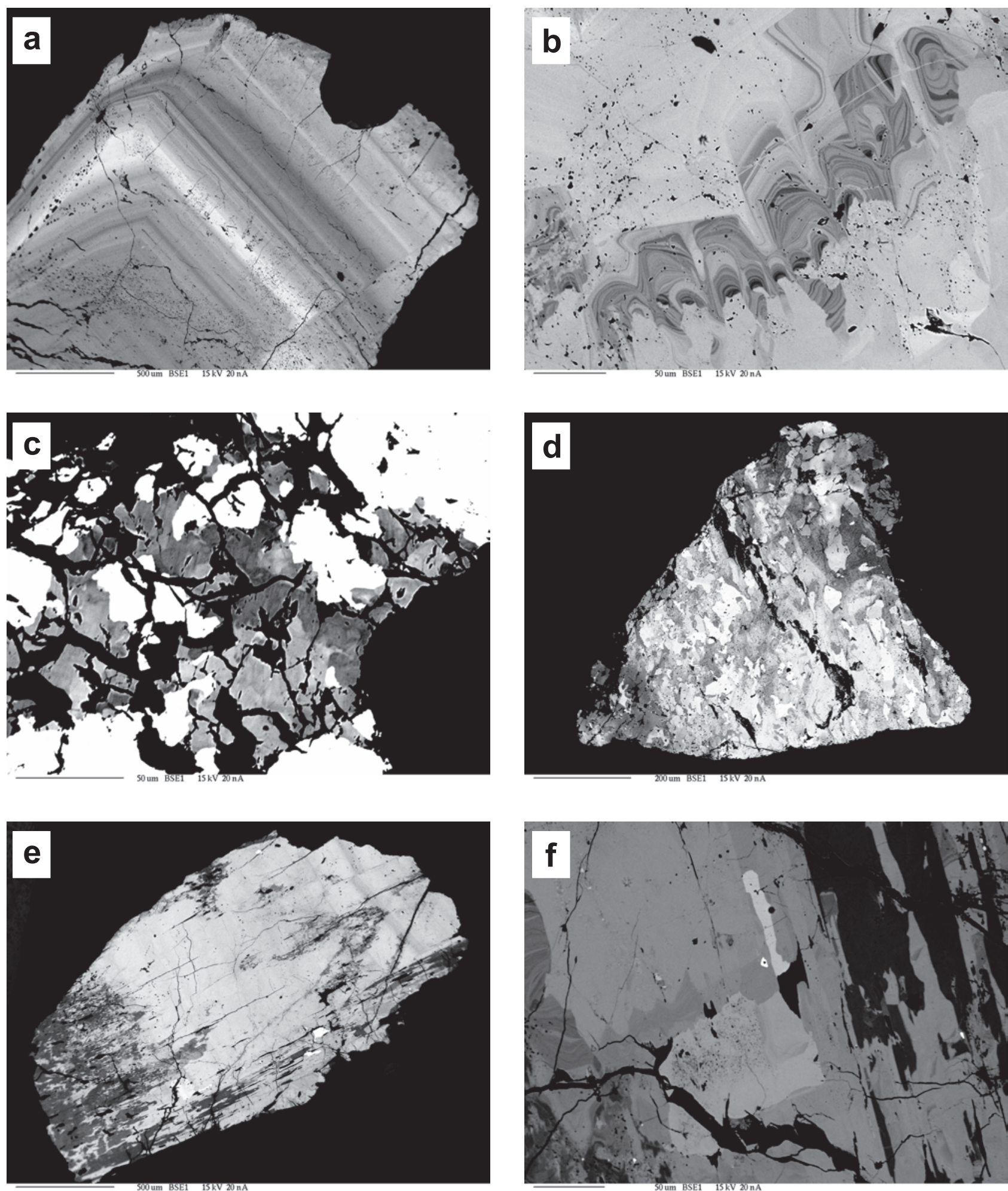

Fig. 2 BSE images of Nb-Ta minerals from Limbach. a - Ferrocolumbite (Ct I) with regular fine oscillatory zoning. $\mathbf{b}-$ Irregular bended fine oscillatory zoning of ferrocolumbite (Ct IIa) replacing surrounding Ct I. c - Irregular mosaic-like intergrowths of ferrocolumbite-ferrotantalite (Ct IIb, white) with $\mathrm{Ta}>\mathrm{Nb}$-rich rutile (dark grey). $\mathbf{d}$ - Irregular mosaic-like intergrowths of ferrotantalite-manganotantalite (Ct IIc, grey) with ferrotapiolite (white). e - Irregular patchy zoning of ferrocolumbite (Ct IId, dark grey) replacing Ct I (pale grey). $\mathbf{f}$ - Sn-rich ixiolite (pale grey) and uraninite (white) inclusions in Ct IId (grey). 
Tab. 1 Representative compositions of columbite-tantalite (Fe-col: ferrocolumbite, Mn-col: manganocolumbite, Fe-tan: ferrotantalite, Mn-tan: manganotantalite) of type I and IIa to IId, ferrotapiolite (Ft) and Sn-rich ixiolite (Ix) from Limbach (in wt. \%).

\begin{tabular}{|c|c|c|c|c|c|c|c|c|}
\hline Mineral & Fe-col & Mn-col & $\mathrm{Fe}-\mathrm{col}$ & Fe-tan & Mn-tan & Fe-tan & $\mathrm{Ft}$ & $\overline{\mathrm{Ix}}$ \\
\hline Type & $\mathrm{Ct} \mathrm{I}$ & $\mathrm{Ct} \mathrm{I}$ & Ct IIa & $\mathrm{Ct} \mathrm{IIb}$ & $\mathrm{Ct}$ IIc & Ct IId & & \\
\hline Anal.\# & B6.1 & C6.3 & B8.10 & D1.8 & D9b9 & $\mathrm{C} 5.12$ & $\mathrm{C} 5.2$ & C5.8 \\
\hline $\mathrm{WO}_{3}$ & 0.11 & 0.10 & 0.00 & 1.96 & 0.00 & 0.26 & 0.01 & 0.12 \\
\hline $\mathrm{Nb}_{2} \mathrm{O}_{5}$ & 49.58 & 34.69 & 42.69 & 24.96 & 10.35 & 28.74 & 6.08 & 9.05 \\
\hline $\mathrm{Ta}_{2} \mathrm{O}_{5}$ & 28.71 & 46.21 & 35.82 & 52.18 & 72.85 & 53.24 & 77.10 & 65.19 \\
\hline $\mathrm{TiO}_{2}$ & 0.80 & 0.77 & 0.82 & 2.78 & 1.05 & 0.30 & 0.44 & 0.35 \\
\hline $\mathrm{ZrO}_{2}$ & 0.47 & 0.52 & 0.19 & 0.30 & 0.51 & 0.09 & 0.18 & 1.63 \\
\hline $\mathrm{SnO}_{2}$ & 0.03 & 0.08 & 0.03 & 0.10 & 0.18 & 0.09 & 0.80 & 9.41 \\
\hline $\mathrm{UO}_{2}$ & 0.07 & 0.25 & 0.05 & 0.18 & 0.10 & 0.03 & 0.00 & 0.09 \\
\hline $\mathrm{Sc}_{2} \mathrm{O}_{3}$ & 0.00 & 0.00 & 0.00 & 0.00 & 0.00 & 0.00 & 0.00 & 0.00 \\
\hline $\mathrm{Y}_{2} \mathrm{O}_{3}$ & 0.00 & 0.00 & 0.00 & 0.00 & 0.00 & 0.00 & 0.00 & 0.00 \\
\hline $\mathrm{Sb}_{2} \mathrm{O}_{3}$ & 0.06 & 0.03 & 0.04 & 0.06 & 0.06 & 0.05 & 0.08 & 0.09 \\
\hline $\mathrm{Fe}_{2} \mathrm{O}_{3}$ calc. & 1.69 & 1.02 & 1.91 & 1.64 & 0.20 & 0.87 & 0.65 & 1.19 \\
\hline $\mathrm{FeO}$ & 11.68 & 7.40 & 13.29 & 10.39 & 4.84 & 8.29 & 13.00 & 6.98 \\
\hline $\mathrm{MnO}$ & 5.44 & 8.89 & 3.01 & 4.34 & 9.53 & 7.66 & 0.83 & 5.37 \\
\hline $\mathrm{MgO}$ & 0.00 & 0.00 & 0.01 & 0.14 & 0.00 & 0.00 & 0.00 & 0.00 \\
\hline $\mathrm{CaO}$ & 0.01 & 0.01 & 0.00 & 0.02 & 0.03 & 0.01 & 0.02 & 0.04 \\
\hline $\mathrm{ZnO}$ & 0.05 & 0.00 & 0.07 & 0.01 & 0.00 & 0.01 & 0.00 & 0.05 \\
\hline $\mathrm{PbO}$ & 0.25 & 0.11 & 0.23 & 0.06 & 0.02 & 0.10 & 0.02 & 0.04 \\
\hline Total & 98.95 & 100.08 & 98.16 & 99.12 & 99.72 & 99.74 & 99.21 & 99.60 \\
\hline \multicolumn{9}{|c|}{ Formulae based on 6 oxygen atoms, 3 cations and $\mathrm{Fe}^{3+} / \mathrm{Fe}^{2+}$ charge-balancing } \\
\hline $\mathrm{W}$ & 0.002 & 0.002 & 0.000 & 0.036 & 0.000 & 0.005 & 0.000 & 0.002 \\
\hline $\mathrm{Nb}$ & 1.434 & 1.076 & 1.286 & 0.803 & 0.370 & 0.928 & 0.225 & 0.322 \\
\hline $\mathrm{Ta}$ & 0.499 & 0.862 & 0.649 & 1.009 & 1.566 & 1.034 & 1.720 & 1.396 \\
\hline $\mathrm{Ti}$ & 0.038 & 0.040 & 0.041 & 0.149 & 0.062 & 0.016 & 0.027 & 0.021 \\
\hline $\mathrm{Zr}$ & 0.015 & 0.017 & 0.006 & 0.010 & 0.020 & 0.003 & 0.007 & 0.063 \\
\hline $\mathrm{Sn}$ & 0.001 & 0.002 & 0.001 & 0.003 & 0.006 & 0.003 & 0.026 & 0.296 \\
\hline Sum B & 1.989 & 1.999 & 1.983 & 2.010 & 2.024 & 1.989 & 2.005 & 2.100 \\
\hline $\mathrm{U}$ & 0.001 & 0.004 & 0.001 & 0.003 & 0.002 & 0.000 & 0.000 & 0.002 \\
\hline $\mathrm{Sc}$ & 0.000 & 0.000 & 0.000 & 0.000 & 0.000 & 0.000 & 0.000 & 0.000 \\
\hline Y & 0.000 & 0.000 & 0.000 & 0.000 & 0.000 & 0.000 & 0.000 & 0.000 \\
\hline $\mathrm{Sb}$ & 0.002 & 0.001 & 0.001 & 0.002 & 0.002 & 0.001 & 0.003 & 0.003 \\
\hline $\mathrm{Fe}^{3+}$ & 0.081 & 0.053 & 0.096 & 0.088 & 0.012 & 0.047 & 0.040 & 0.071 \\
\hline $\mathrm{Fe}^{2+}$ & 0.625 & 0.425 & 0.741 & 0.618 & 0.320 & 0.495 & 0.892 & 0.460 \\
\hline $\mathrm{Mn}$ & 0.295 & 0.517 & 0.170 & 0.262 & 0.638 & 0.464 & 0.058 & 0.358 \\
\hline $\mathrm{Mg}$ & 0.000 & 0.000 & 0.001 & 0.015 & 0.000 & 0.000 & 0.000 & 0.000 \\
\hline $\mathrm{Ca}$ & 0.001 & 0.001 & 0.000 & 0.002 & 0.003 & 0.001 & 0.002 & 0.003 \\
\hline $\mathrm{Zn}$ & 0.002 & 0.000 & 0.003 & 0.001 & 0.000 & 0.001 & 0.000 & 0.003 \\
\hline $\mathrm{Pb}$ & 0.004 & 0.002 & 0.004 & 0.001 & 0.000 & 0.002 & 0.000 & 0.001 \\
\hline Sum A & 1.011 & 1.003 & 1.017 & 0.992 & 0.977 & 1.011 & 0.995 & 0.901 \\
\hline $\operatorname{Sum} A+B$ & 3.000 & 3.002 & 3.000 & 3.002 & 3.001 & 3.000 & 3.000 & 3.001 \\
\hline $\mathrm{Mn} /(\mathrm{Mn}+\mathrm{Fe})$ & 0.29 & 0.52 & 0.17 & 0.27 & 0.66 & 0.46 & 0.06 & 0.40 \\
\hline $\mathrm{Ta} /(\mathrm{Ta}+\mathrm{Nb})$ & 0.26 & 0.44 & 0.34 & 0.56 & 0.81 & 0.53 & 0.88 & 0.81 \\
\hline
\end{tabular}


Tab. 2 Representative compositions of $\mathrm{Ta}>\mathrm{Nb}$ rutile (Rt) from Limbach (in wt. \%).

\begin{tabular}{|c|c|c|c|}
\hline Mineral & $\mathrm{Rt}$ & Rt & $\overline{\mathrm{Rt}}$ \\
\hline Anal.\# & D1.4 & D1.3 & D1.7 \\
\hline $\mathrm{WO}_{3}$ & 0.00 & 0.07 & 0.05 \\
\hline $\mathrm{Nb}_{2} \mathrm{O}_{5}$ & 6.57 & 4.65 & 3.86 \\
\hline $\mathrm{Ta}_{2} \mathrm{O}_{5}$ & 44.32 & 42.16 & 40.86 \\
\hline $\mathrm{TiO}_{2}$ & 38.01 & 43.14 & 45.46 \\
\hline $\mathrm{ZrO}_{2}$ & 0.20 & 0.05 & 0.07 \\
\hline $\mathrm{SnO}_{2}$ & 0.34 & 0.24 & 0.34 \\
\hline $\mathrm{UO}_{2}$ & 0.00 & 0.00 & 0.02 \\
\hline $\mathrm{Sc}_{2} \mathrm{O}_{3}$ & 0.00 & 0.00 & 0.00 \\
\hline $\mathrm{Y}_{2} \mathrm{O}_{3}$ & 0.00 & 0.00 & 0.00 \\
\hline $\mathrm{Sb}_{2} \mathrm{O}_{3}$ & 0.06 & 0.03 & 0.06 \\
\hline $\mathrm{Fe}_{2} \mathrm{O}_{3}$ calc. & 2.39 & 1.29 & 2.05 \\
\hline $\mathrm{FeO}$ & 7.65 & 7.37 & 6.56 \\
\hline $\mathrm{MnO}$ & 0.20 & 0.15 & 0.17 \\
\hline $\mathrm{MgO}$ & 0.00 & 0.00 & 0.00 \\
\hline $\mathrm{CaO}$ & 0.02 & 0.01 & 0.02 \\
\hline $\mathrm{ZnO}$ & 0.00 & 0.00 & 0.00 \\
\hline $\mathrm{PbO}$ & 0.04 & 0.03 & 0.01 \\
\hline Total & 99.80 & 99.19 & 99.53 \\
\hline \multicolumn{4}{|c|}{$\begin{array}{l}\text { Formulae based on } 2 \text { oxygen atoms, } \\
1 \text { cation and } \mathrm{Fe}^{3+} / \mathrm{Fe}^{2+} \text { charge-balancing }\end{array}$} \\
\hline W & 0.000 & 0.000 & 0.000 \\
\hline $\mathrm{Nb}$ & 0.057 & 0.039 & 0.032 \\
\hline $\mathrm{Ta}$ & 0.231 & 0.215 & 0.204 \\
\hline $\mathrm{Ti}$ & 0.547 & 0.607 & 0.628 \\
\hline $\mathrm{Zr}$ & 0.002 & 0.000 & 0.001 \\
\hline $\mathrm{Sn}$ & 0.003 & 0.002 & 0.002 \\
\hline $\mathrm{U}$ & 0.000 & 0.000 & 0.000 \\
\hline $\mathrm{Sc}$ & 0.000 & 0.000 & 0.000 \\
\hline Y & 0.000 & 0.000 & 0.000 \\
\hline $\mathrm{Sb}$ & 0.000 & 0.000 & 0.000 \\
\hline $\mathrm{Fe}^{3+}$ & 0.034 & 0.018 & 0.028 \\
\hline $\mathrm{Fe}^{2+}$ & 0.122 & 0.115 & 0.101 \\
\hline $\mathrm{Mn}$ & 0.003 & 0.002 & 0.003 \\
\hline $\mathrm{Mg}$ & 0.000 & 0.000 & 0.000 \\
\hline $\mathrm{Ca}$ & 0.000 & 0.000 & 0.000 \\
\hline $\mathrm{Zn}$ & 0.000 & 0.000 & 0.000 \\
\hline $\mathrm{Pb}$ & 0.000 & 0.000 & 0.000 \\
\hline Sum & 0.999 & 0.998 & 0.999 \\
\hline $\mathrm{Mn} /(\mathrm{Mn}+\mathrm{Fe})$ & 0.02 & 0.01 & 0.02 \\
\hline $\mathrm{Ta} /(\mathrm{Ta}+\mathrm{Nb})$ & 0.80 & 0.85 & 0.86 \\
\hline
\end{tabular}

Four textural and paragenetic patterns can be distinguished in Ct II (Tab. 1, Figs 2b-f and 3): $\boldsymbol{C} \boldsymbol{t}$ II $\boldsymbol{a}$ forms irregular bended zones of ferrocolumbite (up to $0.3 \mathrm{~mm}$ long), with fine oscillatory zoning, which replaces $\mathrm{Ct} \mathrm{I}$. The zoning is caused by $\mathrm{Nb}-\mathrm{Ta}$ variations; $\mathrm{Mn} /(\mathrm{Mn}+\mathrm{Fe})=0.16-0.19$ and $\mathrm{Ta} /(\mathrm{Ta}+\mathrm{Nb})=0.16-0.34$. Ct IIb occurs as irregular patchy intergrowths of ferrocolumbite-ferrotantalite with
Tab. 3 Representative compositions of uranmicrolite (Umc) to microlite (Mcr) from Limbach (wt. \%).

\begin{tabular}{|c|c|c|c|c|}
\hline $\begin{array}{l}\text { Mineral } \\
\text { Anal.\# }\end{array}$ & $\begin{array}{l}\text { Umc } \\
\text { B8.1 }\end{array}$ & $\begin{array}{l}\text { Umc } \\
\text { B8.2 }\end{array}$ & $\begin{array}{l}\text { Umc } \\
\text { B8.3 }\end{array}$ & $\begin{array}{r}\text { Mcr } \\
\text { D9b10 }\end{array}$ \\
\hline $\mathrm{WO}_{3}$ & 0.00 & 0.00 & 0.31 & 0.00 \\
\hline $\mathrm{Nb}_{2} \mathrm{O}_{5}$ & 6.99 & 6.55 & 7.77 & 2.82 \\
\hline $\mathrm{Ta}_{2} \mathrm{O}_{5}$ & 61.89 & 60.19 & 57.09 & 70.61 \\
\hline $\mathrm{TiO}_{2}$ & 2.51 & 2.81 & 2.11 & 1.52 \\
\hline $\mathrm{ZrO}_{2}$ & 0.00 & 0.12 & 0.06 & 0.18 \\
\hline $\mathrm{SnO}_{2}$ & 0.14 & 0.16 & 0.07 & 0.17 \\
\hline $\mathrm{UO}_{2}$ & 13.05 & 15.48 & 19.34 & 8.63 \\
\hline $\mathrm{Sc}_{2} \mathrm{O}_{3}$ & 0.00 & 0.00 & 0.00 & 0.00 \\
\hline $\mathrm{Y}_{2} \mathrm{O}_{3}$ & 0.00 & 0.00 & 0.00 & 0.00 \\
\hline $\mathrm{Sb}_{2} \mathrm{O}_{3}$ & 0.07 & 0.06 & 0.19 & 0.31 \\
\hline $\mathrm{FeO}$ & 0.32 & 0.25 & 0.43 & 4.90 \\
\hline $\mathrm{MnO}$ & 0.11 & 0.05 & 0.07 & 0.46 \\
\hline $\mathrm{MgO}$ & 0.00 & 0.00 & 0.00 & 0.00 \\
\hline $\mathrm{CaO}$ & 6.92 & 6.34 & 5.69 & 4.61 \\
\hline $\mathrm{ZnO}$ & 0.00 & 0.00 & 0.00 & 0.01 \\
\hline $\mathrm{PbO}$ & 0.23 & 0.20 & 0.25 & 0.20 \\
\hline $\mathrm{H}_{2} \mathrm{O}$ calc. & 1.64 & 1.61 & 1.55 & 1.63 \\
\hline Total & 93.87 & 93.82 & 94.93 & 96.05 \\
\hline \multicolumn{5}{|c|}{ Formulae based on $\mathrm{B}=2$ cations and $1 \mathrm{OH}$ anion } \\
\hline $\mathrm{W}$ & 0.000 & 0.000 & 0.008 & 0.000 \\
\hline $\mathrm{Nb}$ & 0.289 & 0.275 & 0.339 & 0.117 \\
\hline $\mathrm{Ta}$ & 1.538 & 1.523 & 1.497 & 1.770 \\
\hline $\mathrm{Ti}$ & 0.173 & 0.197 & 0.153 & 0.105 \\
\hline $\mathrm{Zr}$ & 0.000 & 0.005 & 0.003 & 0.008 \\
\hline Sum $B$ & 2.000 & 2.000 & 2.000 & 2.000 \\
\hline $\mathrm{Sn}$ & 0.005 & 0.006 & 0.003 & 0.006 \\
\hline $\mathrm{U}$ & 0.265 & 0.320 & 0.415 & 0.177 \\
\hline $\mathrm{Sc}$ & 0.000 & 0.000 & 0.000 & 0.000 \\
\hline $\mathrm{Y}$ & 0.000 & 0.000 & 0.000 & 0.000 \\
\hline $\mathrm{Sb}$ & 0.003 & 0.002 & 0.008 & 0.012 \\
\hline $\mathrm{Fe}^{2+}$ & 0.024 & 0.019 & 0.035 & 0.378 \\
\hline $\mathrm{Mn}$ & 0.009 & 0.004 & 0.006 & 0.036 \\
\hline $\mathrm{Mg}$ & 0.000 & 0.000 & 0.000 & 0.000 \\
\hline $\mathrm{Ca}$ & 0.678 & 0.632 & 0.588 & 0.455 \\
\hline $\mathrm{Zn}$ & 0.000 & 0.000 & 0.000 & 0.001 \\
\hline $\mathrm{Pb}$ & 0.006 & 0.005 & 0.006 & 0.005 \\
\hline $\operatorname{Sum} A$ & 0.990 & 0.988 & 1.061 & 1.070 \\
\hline $\mathrm{O}$ & 6.675 & 6.715 & 6.908 & 6.701 \\
\hline $\mathrm{OH}$ & 1.000 & 1.000 & 1.000 & 1.000 \\
\hline$\% \mathrm{U}$ in $A$-site & 26.77 & 32.39 & 39.11 & 16.54 \\
\hline $\mathrm{Ta} /(\mathrm{Ta}+\mathrm{Nb})$ & 0.84 & 0.85 & 0.82 & 0.94 \\
\hline
\end{tabular}

Ta-rich rutile $(c .1 \mathrm{~mm}$ in size $) ; \mathrm{Mn} /(\mathrm{Mn}+\mathrm{Fe})=0.19-0.27$ and $\mathrm{Ta} /(\mathrm{Ta}+\mathrm{Nb})=0.49-0.57$. Ct IIb shows increased $\mathrm{Ti}$ and $\mathrm{W}$ contents (up to $2.3 \mathrm{wt} . \% \mathrm{TiO}_{2}$ and $0.7 \mathrm{wt} \%$ $\mathrm{WO}_{3}$ ). $\boldsymbol{C t}$ IIc forms irregular mosaic intergrowths of ferrotantalite-manganotantalite with ferrotapiolite (c. $0.6 \mathrm{~mm}$ 


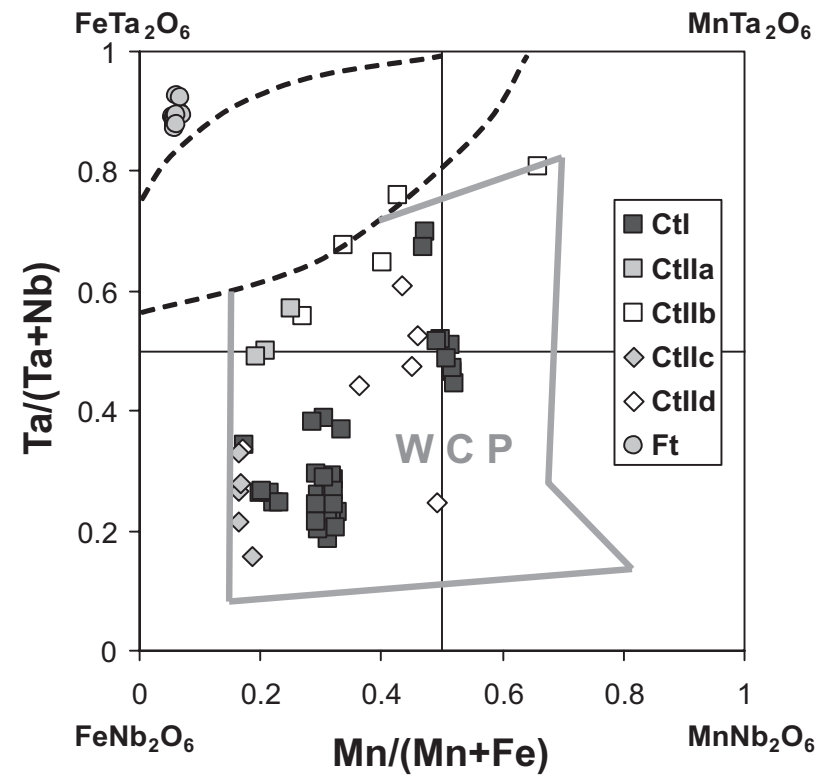

Fig. 3 Quadrilateral diagram (atomic proportions) of columbite-tantalite types (Ct I, Ct IIa to IId) and ferrotapiolite (Ft) from Limbach. Field of columbite-tantalite compositions from the West-Carpathian granitic pegmatites (WCP) is shown for comparison (Uher et al. 1994, 1998; Uher and Benko 1997; Uher 2000; Novák et al. 2000, unpublished data of P. U.).

in size $) ; \mathrm{Mn} /(\mathrm{Mn}+\mathrm{Fe})=0.34-0.66$ and $\mathrm{Ta} /(\mathrm{Ta}+\mathrm{Nb})=$ 0.65-0.81. Ct IId occurs as irregular patchy zones of ferrocolumbite to ferrotantalite $(0.2-0.9 \mathrm{~mm})$, mainly along cracks and rims of $\mathrm{Ct} \mathrm{I} ; \mathrm{Mn} /(\mathrm{Mn}+\mathrm{Fe})=0.43-0.49$ and $\mathrm{Ta} /(\mathrm{Ta}+\mathrm{Nb})=0.24-0.61$ (Tab. 1, Figs 3 and 4).

Despite textural variability and locally specific compositions, all types of Ct II show a chemistry generally similar to $\mathrm{Ct}$ I with both large variations in $\mathrm{Mn} / \mathrm{Fe}$ as well as $\mathrm{Ta} / \mathrm{Nb}$ ratio: $\mathrm{Mn} /(\mathrm{Mn}+\mathrm{Fe})=0.17-0.66$ and $\mathrm{Ta} /(\mathrm{Ta}+$ $\mathrm{Nb})=0.16-0.81$ (Tab. 1, Figs 3 and 4).

Ferrotapiolite forms anhedral to subhedral intergrowths with Ct IIc, 60 to $100 \mu \mathrm{m}$ in size (Fig. 2d). The ferrotapiolite shows relatively consistent compositions with $\mathrm{Mn} /(\mathrm{Mn}+\mathrm{Fe})=0.05-0.07$ and $\mathrm{Ta} /(\mathrm{Ta}+\mathrm{Nb})=$ 0.87-0.93. Titanium and $\mathrm{Sn}$ contents are relatively low, 0.3 to 1.4 wt. $\% \mathrm{TiO}_{2}$ and 0.3 to 0.8 wt. $\% \mathrm{SnO}_{2}$, respectively (Tab. 1, Figs 3 and 4).

The $\mathrm{Ta}>\mathrm{Nb}$-rich rutile (strüverite) occurs as anhedral irregular mosaic intergrowths with $\mathrm{Ct}$ IIb, up to 200 $\mu \mathrm{m}$ across (Fig. 2c). Despite their irregular zoning due to some $\mathrm{Nb} / \mathrm{Ta}$ variations, strüverite is compositionally relatively uniform with $\mathrm{Mn} /(\mathrm{Mn}+\mathrm{Fe})=0.01-0.03$ and $\mathrm{Ta} /(\mathrm{Ta}+\mathrm{Nb})=0.80-0.84$, without increased contents of any other elements (Tab. 2, Figs 3 and 4).

In one case a Sn-rich ixiolite-like phase was found as $110 \mu \mathrm{m}$ irregular inclusion in Ct IId in vicinity of uraninite inclusion (Fig. 2f). This Sn-rich ixiolite contains 9.4 wt. $\% \mathrm{SnO}_{2}(0.3 \mathrm{Sn}$ apfu $)$ with $\mathrm{Mn} /(\mathrm{Mn}+\mathrm{Fe})=0.40$ and $\mathrm{Ta} /(\mathrm{Ta}+\mathrm{Nb})=0.81$. Ixiolite reveals slightly increased $\mathrm{Zr}$ contents $\left(1.5-1.6\right.$ wt. $\left.\% \mathrm{ZrO}_{2}\right)$, but very low $\mathrm{W}$ and $\mathrm{Ti}$ concentrations, up to 0.4 wt. $\% \mathrm{TiO}_{2}$ and 0.1 wt. $\% \mathrm{WO}_{3}$, respectively (Tab. 1, Figs 3 and 4).

Rare uranmicrolite to microlite forms anhedral, $5-15 \mu \mathrm{m}$ thick overgrowths on uraninite in patchy zoned Ct IId (Fig. 5a). Characteristic of this material are $\mathrm{Ta} /(\mathrm{Ta}+\mathrm{Nb})=0.82-0.94, \mathrm{U}=0.18-0.42 \mathrm{apfu}$, low contents of $\mathrm{Ti}\left(1.5-2.8\right.$ wt. \% $\mathrm{TiO}_{2}, 0.1-0.2 \mathrm{Ti}$ apfu $)$ and $\mathrm{Pb}(0.2-1$ wt. \% $\mathrm{PbO}, 0.005-0.02 \mathrm{~Pb}$ apfu); contents of $\mathrm{W}, \mathrm{Zr}, \mathrm{Sn}$ and $\mathrm{Sb}$ are negligible (Tab. 3, Figs 3 and 4). Elsewhere, a single anhedral uraninite inclusion was found in Ct II (Fig. 2f).
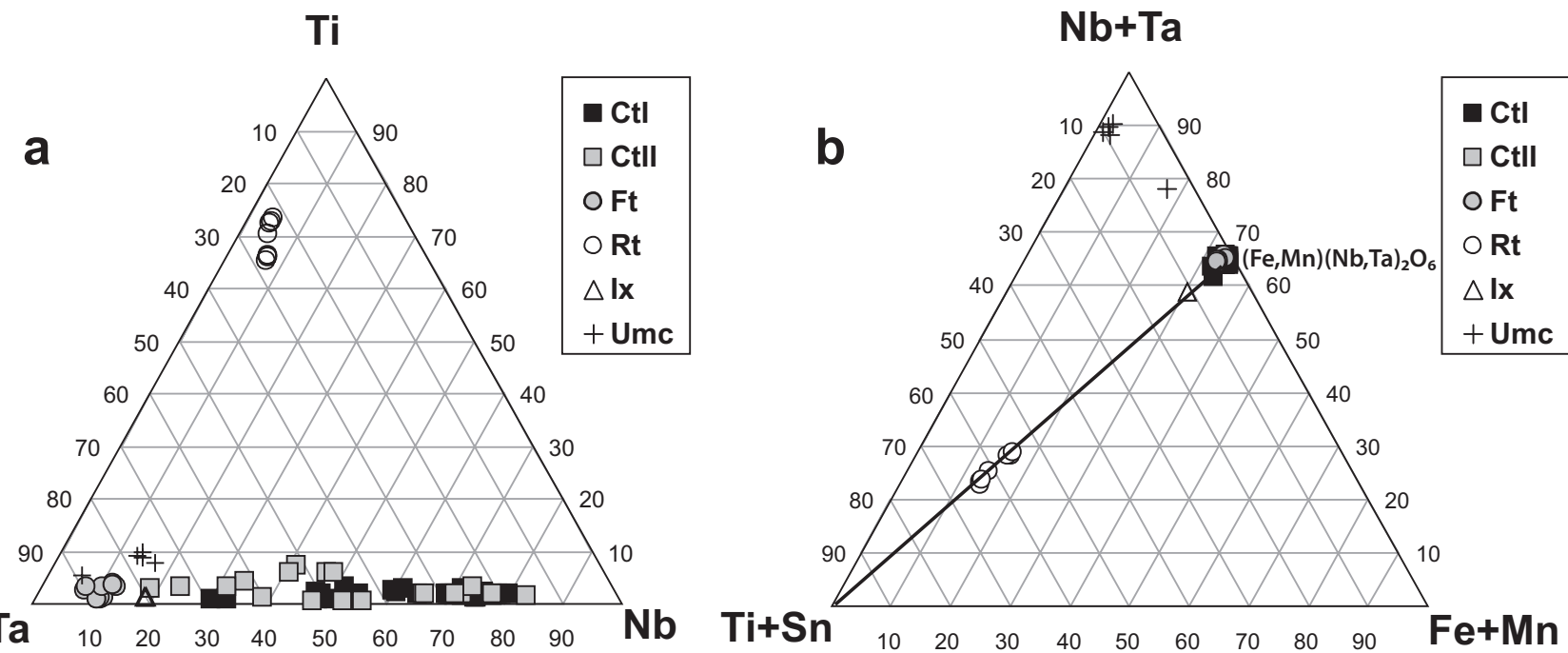

Fig. 4 Triangular diagrams of Nb-Ta minerals from Limbach (atomic proportions). $\mathbf{a}-\mathrm{Ti}-\mathrm{Ta}-\mathrm{Nb}$ diagram. $\mathbf{b}-(\mathrm{Nb}+\mathrm{Ta})-(\mathrm{Ti}+\mathrm{Sn})-(\mathrm{Fe}+\mathrm{Mn})$ diagram. CtI: columbite-tantalite I, CtII: columbite-tantalite II, Ft: ferrotapiolite, Rt: rutile, Ix: ixiolite, Umc: uranmicrolite to microlite. 

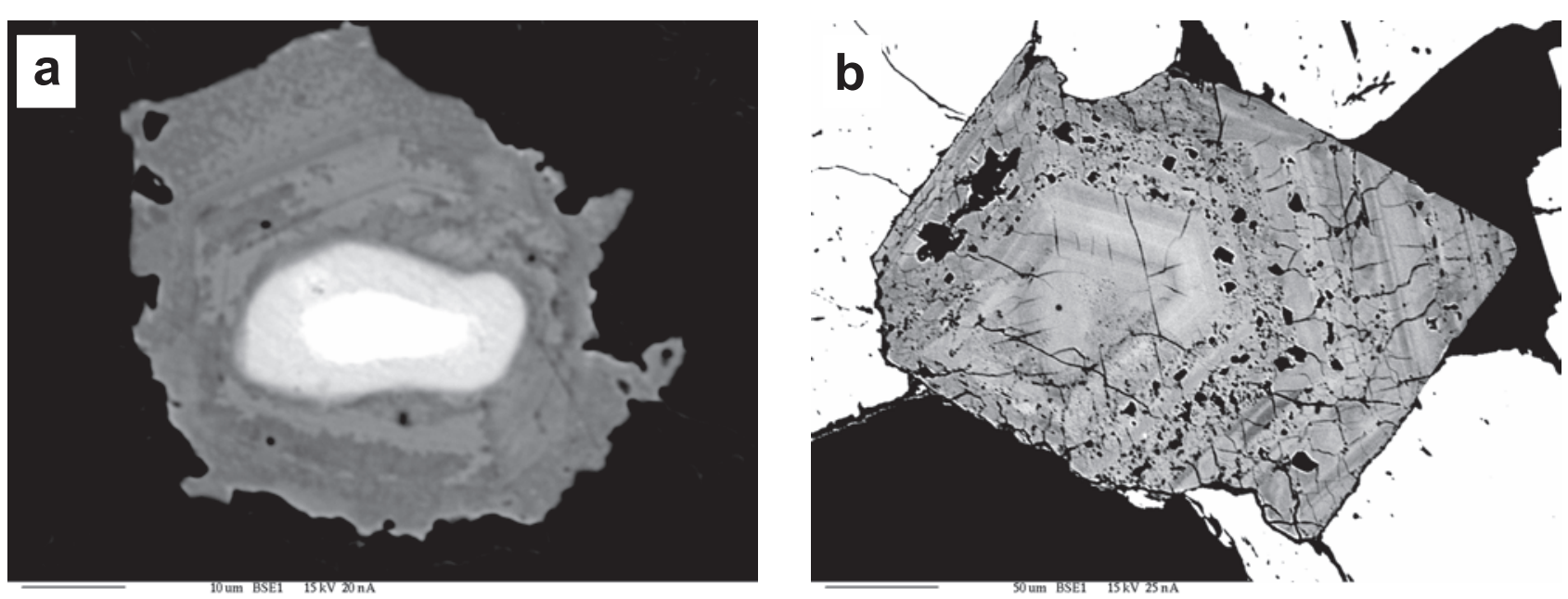

Fig. 5 BSE images of inclusions in columbite-tantalite (Ct IId) from Limbach. a - oscillatory to irregular zoned overgrowths of uranmicrolite to microlite (grey) on uraninite (white to pale grey core) in Ct IId (black). b - partly metamict zircon with regular oscillatory zoning (grey) in Ct IId (white).

In addition to the above-mentioned $\mathrm{Nb}$-Ta oxide minerals, euhedral to subhedral zircon inclusions $c .250 \mu \mathrm{m}$ in size occur in columbite-tantalite (Ct IId) (Fig. 5b). Zircon exhibits regular fine oscillatory zoning with some probably (partly) metamict zones, rich in cracks. The zircon shows high $\mathrm{Hf}, \mathrm{U}$, Th, and $\mathrm{P}$ contents (11.6-14.6 wt. $\% \mathrm{HfO}_{2}, 0.3-2.6$ wt. $\% \mathrm{UO}_{2}, 0.0-3.0$ wt. $\% \mathrm{Y}_{2} \mathrm{O}_{3}$, and $0.0-3.6$ wt. $\% \mathrm{P}_{2} \mathrm{O}_{5}$, respectively). Locally, anhedral inclusions of quartz and muscovite in columbite-tantalite are also present.

\section{Discussion and conclusion}

\subsection{Comparison to regional Nb-Ta assem- blages}

The $\mathrm{Nb}$-Ta minerals were found only in recent alluvial placers of the Limbach Brook, not in a primary host-rock in situ. Nevertheless, regional geology as well as overall paragenetic, textural, and compositional character of the assemblage suggest rare-element granitic pegmatite(s) as the parental rock of the $\mathrm{Nb}$-Ta mineralization. It is questionable whether the $\mathrm{Nb}$-Ta alluvial minerals originated from one large pegmatite vein or several pegmatite bodies. The Limbach locality is situated in the region of the BGM, Hercynian orogenic S-type granites with numerous dikes of related pegmatites. The granitic rocks alone belong to a common plutonic suite with low to moderate contents of rare lithophile elements (such as $\mathrm{Nb}, \mathrm{Ta}, \mathrm{Sn}, \mathrm{Li}, \mathrm{Rb}, \mathrm{Cs}, \mathrm{Be}$ and $\mathrm{B}$ ), lacking any evidence for extreme magmatic fractionation or possible concentrations of rare-element mineralization (Cambel and Vilinovič 1987; Petrík et al. eds 2001). On the other hand, the most fractionated granitic pegmatites of the BGM contain accessory beryl and $\mathrm{Nb}$-Ta phases, namely fer- rocolumbite to ferrotantalite, rarely manganocolumbite. Their chemical composition is similar to the Limbach columbite-tantalite (Bratislava, Kamzík and Bratislava, Patrónka pegmatites; Uher et al. 1994). However, the Limbach locality represents a more fractionated $\mathrm{Nb}-\mathrm{Ta}$ assemblage with Mn, Ta-rich (manganotantalite, Sn-rich ixiolite) and Ta-rich members (ferrotapiolite, Ta-rich rutile, uranmicrolite to microlite) in comparison to the other known pegmatites of the BGM. Such relatively large variations in $\mathrm{Mn} / \mathrm{Fe}$ and $\mathrm{Ta} / \mathrm{Nb}$ ratios with $\mathrm{Ta}$, Mn-dominated members resemble the Moravany nad Váhom, Striebornica granitic pegmatite in the adjacent Bojná Granitic Massif, Považský Inovec Mountains. This is the most fractionated pegmatite body in the Western Carpathians, where columbite-tantalite and ferrotapiolite attain $\mathrm{Mn} /(\mathrm{Mn}+\mathrm{Fe})=0.18-0.67$ and $0.05-0.10, \mathrm{Ta} /(\mathrm{Ta}$ $+\mathrm{Nb})=0.09-0.81$ and $0.83-0.90$, respectively (Uher et al. 1994; Uher and Broska 1995; Novák et al. 2000). Generally, compositional range of columbite-tantalite from the Limbach placers is very similar to granitic pegmatites of the Western Carpathian area (Fig. 3). Analogous columbite-tantalite compositions and fractionation trends with increasing $\mathrm{Ta} /(\mathrm{Ta}+\mathrm{Nb})$ and low to moderate $\mathrm{Mn} /(\mathrm{Mn}+\mathrm{Ta})$ ratios exhibit beryl-bearing and $\mathrm{Li}$, F-poor granitic pegmatites, for instance the Greer Lake and PEG Groups, Canada (Černý 1989). On the contrary, the columbite-tantalite fractionation trends commonly reach $\mathrm{Mn}$, Ta-rich members with $\mathrm{Ta} /(\mathrm{Ta}+\mathrm{Nb})$ and $\mathrm{Mn} /(\mathrm{Mn}+$ $\mathrm{Fe})>0.8$ in the most fractionated beryl-type pegmatites (e.g., Separation Lake, Canada - Tindle and Breaks 2000; Scheibengraben, Maršíkov, Czech Republic - Novák et al. 2003) and Li, F-rich complex-type of the rare-element granitic pegmatites (Černý 1989).

Based on the above-mentioned data, the $\mathrm{Nb}$-Ta oxide minerals from Limbach should have originated in an evolved granitic pegmatite environment, most prob- 
ably analogous to the rare-element class, LCT family, beryl-columbite subtype (sensu Černý and Ercit 2005 classification).

In addition, Hf-rich, partly metamict zircon from Limbach is also characteristic of evolved, rare-element granitic pegmatites and its composition is analogous to zircon from granitic pegmatites of the beryl-columbite subtype from the BGM and other evolved pegmatite populations in the Western Carpathians (Uher and Černý 1998).

\subsection{Evolution of $\mathrm{Nb}$-Ta phases}

Textural patterns indicate two main crystallization events in the Limbach columbite-tantalite (Fig. 2): (1) older primary magmatic solidification of $\mathrm{Ct}$ I with regular fine to coarse oscillatory, rarely diffuse compositional zoning, and (2) post-magmatic recrystallization of Ct II with irregular bended fine-oscillatory, patchy to mosaic zoning (IIa to IId). The regular oscillatory zoning reflects the growth of columbite-tantalite parallel to their crystallographic planes and it is generally considered as a result of magmatic crystallization. On the other hand, irregular, bended, patchy, or mosaic textures with anhedral boundaries indicate non-magmatic, metamorphic or hydrothermal recrystallization, partial leaching and replacement or alteration phenomena (e.g. Lahti 1987; Baldwin 1989; Černý et al. 1992; Tindle and Breaks 1998, 2000). We interpret all these irregular textures of the Limbach columbites-tantalites as products of subsolidus partial leaching and subsequent replacement and recrystallization of the host granitic-pegmatitic rocks during post-magmatic uplift and retrograde fluid overprint. On the basis of textural relations, the origin of the anhedral mosaic of ferrotapiolite, $\mathrm{Ta}>\mathrm{Nb}$ rutile, $\mathrm{Sn}$-rich ixiolite and uranmicrolite to microlite is closely connected with this subsolidus overprint. However, at least ferrotapiolite and possibly also rutile could have initially originated as primary magmatic phases together with $\mathrm{Ct}$ I, but they were probably completely recrystallized during the subsolidus event. On the contrary, euhedral shape and regular oscillation zoning of zircon indicate its primary magmatic origin, possibly with subsequent partial metamictization (Fig. 5b).

The primary evolutional trend of columbite-tantalite (Ct I) reveals a common progression from ferrocolumbite to manganotantalite composition, with relatively continuous increase in both the $\mathrm{Mn} / \mathrm{Fe}$ and $\mathrm{Ta} / \mathrm{Nb}$ ratios $[\mathrm{Mn} /(\mathrm{Mn}+\mathrm{Fe})=0.17$ to 0.52 and $\mathrm{Ta} /(\mathrm{Ta}+\mathrm{Nb})=0.19$ to 0.70 ; Fig. 3]. Such an evolutionary trend indicates a moderate degree of fractionation with low F activity, analogous mainly to the beryl-columbite subtype of rare-element granitic pegmatites (e.g., Černý et al. 1986; Černý 1989; Tindle and Breaks 2000; Novák et al. 2003;
Galliski and Černý 2006). In general, the compositional similarity of post-magmatic Ct II and Ct I together with (re)crystallization of Ta-rich phases indicates recrystallization of primary magmatic assemblage without any external influx of metallic ions or different fluids (e.g., F, B and/or P-rich).

Acknowledgements The authors thank P. Konečný for assistance with the electron microprobe. We are also grateful to M. Novák, L. Groat and V. Janoušek for their constructive comments and reviews of the manuscript. This work was supported by the Slovak Research and Development Agency under the contract No. APVV0557-06.

\section{References}

Bagdasaryan GP, Gukasyan RC, Cambel B, Veselský J (1982) The age of Malé Karpaty Mts. granitoid rocks determined by Rb-Sr isochrone method. Geol Zbor Geol Carpath 33: 131-140

Bakos F, Chovan M (eds) (2004) Gold in Slovakia. Slovenský Skauting, Bratislava, pp 1-298

BALDWIN JR (1989) Replacement phenomena in tantalum minerals from rare-metal pegmatites in South Africa and Namibia. Mineral Mag 53: 571-581

Cambel B, Vilinovič V (1987) Geochemistry and petrology of the granitoid rocks of the Malé Karpaty Mts. (in Slovak). Veda, Bratislava, pp 1-248

ČERNÝ P (1989) Characteristics of pegmatite deposits of tantalum. In: Möller P, Černý P, Saupé F (eds) Lanthanides, Tantalum and Niobium. Springer-Verlag, Berlin, pp 192-236

ČERNÝ P, ERCIT TS (1989) Mineralogy of niobium and tantalum: crystal chemical relationships, paragenetic aspects and their economic implications. In: Möller P, Černý P, Saupé F (eds) Lanthanides, Tantalum and Niobium. Springer-Verlag, Berlin, pp 27-79

ČERNÝ P, ERCIT TS (2005) The classification of granitic pegmatites revisited. Canad Mineral 43: 2005-2026

Černý P, Meintzer RE, Anderson AJ (1985) Extreme fractionaction in rare-element granitic pegmatites: selected examples of data and mechanisms. Canad Mineral 23: 381-421

Černý P, Goad BE, Hawthorne FC, Chapman R (1986) Fractionaction trends of the $\mathrm{Nb}$ - and Ta-bearing oxide minerals in the Greer Lake pegmatitic granite and its pegmatite aureole, southeastern Manitoba. Amer Miner 71: 501-517

ČERnÝ P, NovÁk M, Chapman R (1992) Effects of sillimanite-grade metamorphism and shearing on $\mathrm{Nb}$-Ta oxide minerals in granitic pegmatites: Maršíkov, northern Moravia, Czechoslovakia. Canad Mineral 30: 699-718 
DÁvidová Š (1970) Characteristics of the Malé Karpaty pegmatites (in German). Geol Zbor Geol Carpath 21: $115-137$

DÁvidové Š (1978) Mineralogy and petrography of the pegmatites in the Tatrides crystalline complex (in Slovak). Miner Slov 10: 127-146

GALLISKI MA, ČERNÝ P (2006) Geochemistry and structural state of columbite-group minerals in granitic pegmatites of the Pampean Ranges, Argentina. Canad Mineral 44: 645-666

Korikovsky SP, CAmbel B, Miklóš J, JanÁk M (1984) Metamorphism of the Malé Karpaty Mts.: stages, zonality, relationship to granitic rocks. Geol Zbor Geol Carpath 35: 437-462 (in Russian)

LAHTI SI (1987) Zoning in columbite-tantalite crystals from the granitic pegmatites of the Eräjärvi area, southern Finland. Geochim Cosmochim Acta 51: 509-517

LinNen RL (1998) The solubility of Nb-Ta-Zr-Hf-W in granitic melts with $\mathrm{Li}$ and $\mathrm{Li}+\mathrm{F}$ : constraints for mineralization in rare metal granites and pegmatites. Econ Geol 93: 1013-1025

LinNen RL, KepPler H (1997) Columbite solubility in granitic melts: consequences for the enrichment and fractionation of $\mathrm{Nb}$ and $\mathrm{Ta}$ in the Earth's crust. Contrib Mineral Petrol 128: 213-227

Novák M, Uher P, ČERnÝ P, Siman P (2000) Compositional variations in ferrotapiolite + tantalite pairs from the beryl-columbite pegmatite at Moravany nad Váhom, Slovakia. Mineral Petrol 69: 295-306

NovÁK M, ČERNÝ P, UHER P (2003) Extreme variation and apparent reversal of $\mathrm{Nb}$-Ta fractionaction in columbitegroup minerals from Scheibengraben beryl-columbite granitic pegmatite, Maršíkov, Czech Republic. Eur J Mineral 15: 565-574
Petrík I, Kohút M, Broska I (eds) (2001) Granitic Plutonism of the Western Carpathians. Guide Book to Eurogranites 2001. Veda, Bratislava, pp 1-116

Tindle AG, Breaks FW (1998) Oxide minerals of the Separation Rapids rare-element granitic pegmatite group, northwestern Ontario. Canad Mineral 36: 609-635

Tindle AG, Breaks FW (2000) Columbite-tantalite mineral chemistry from rare-element granitic pegmatites: Separation Lake area, N.W. Ontario, Canada. Mineral Petrol 70: 165-198

UHER P (2000) Stibiotantalite from the Dúbrava antimony deposit, Slovakia: a product of hydrothermal alteration of columbite-tantalite. Miner Slov 32: 109-114 (in Slovak with English summary)

Uher P, BenKo P (1997) Beryl-columbite pegmatite from the Dúbrava deposit, the Nízke Tatry Mountains (Western Carpathians, Slovakia) . Natura Carpatica 38: 181-184 (in Slovak with English summary)

Uher P, BRoska I (1995) Pegmatites in two suites of Variscan orogenic rocks (Western Carpathians, Slovakia). Mineral Petrol 55: 27-36

Uher P, ČERnÝ P (1998) Accessory zircon in Hercynian granitic pegmatites of the Western Carpathians, Slovakia. Geol Carpath 49: 261-270

Uher P, Černý P, NovÁk M, Siman P (1994) Niobium-tantalum minerals from granitic pegmatites in the Malé Karpaty, Považský Inovec and Žiar Mountains, Western Carpathians, Slovakia. Miner Slov 26: 157-164

Uher P, Černý P, Chapman R, Határ J, Miko O (1998) Evolution of $\mathrm{Nb}$, Ta-oxide minerals in the Prašivá granitic pegmatites, Slovakia. I. Primary Fe, Ti-rich assemblage. Canad Mineral 36: 525-534 